Vicast microresonators are exceptionally smooth, and the scattering component of optical loss is therefore very low (such microresonators are said to be material-loss-limited). Because of these properties, Vahala and co-workers believe that their method "has a secondary application for rapid evaluation of optical loss in previously untested polymers." The researchers reported that with the use of other polymers such as poly(methyl methacrylate), which is known to exhibit even lower material losses than PDMS or Vicast, "replicated devices with $Q$ factors in excess of 100 million, that is, comparable with their masters, could be molded and used to probe nonlinear optical and thermo-optic tuning effects."

STEVEN TROHALAKI

\section{Tunable Superhydrophobic Surfaces Fabricated by Nanosphere Lithography}

Superhydrophobic materials received much attention after the discovery of water-repellent behavior in micro- and nanostructured plant surfaces. In examining the influence of nanostructure on surface water-repellent behavior, J.-Y. Shiu, C.-W. Kuo, and P. Chen of Academia Sinica and C.-Y. Mou of National Taiwan University have fabricated well-ordered, tunable superhydrophobic surfaces with a variable water-contact angle (tuned from $132^{\circ}$ to $170^{\circ}$ ) using nanosphere lithography and oxygen plasma. They accomplished this by creating rough surfaces covered with low-surface-energy molecules and by roughening the surface of hydrophobic materials.

As reported in the February 24 issue of Chemistry of Materials, the scientists obtained single- and double-layer close-packed polystyrene (PS) arrays by spin-coating monodisperse PS bead solutions onto substrates. This approach to nanosphere lithography achieves arrays of self-ordered, close-packed nanostructures. In a first approach, they used 440-nm-diameter PS beads to form single-layer arrays and later reduced the beads to $360 \mathrm{~nm}, 330 \mathrm{~nm}$, and finally $190 \mathrm{~nm}$ by oxygen plasma treatment. The nanosphere separation remained constant during the oxygen plasma treatment. After the oxygen plasma treatment, the arrays were coated with a 20-nm-thick gold film and modified with octadecanethiol (ODT). The apparent water-contact angle of the single-layer surfaces changed monotonically from $132^{\circ}$ (for 440-nmdiameter arrays) to $168^{\circ}$ (for 190-nm-diameter size-reduced arrays).

The water-contact angles on the nanostructured surfaces were much larger than that of an ODT-modified gold surface on a flat substrate $\left(114^{\circ}\right)$. The researchers said that this is an indication that the surface nanostructure governs the superhydrophobic behavior of surfaces.

Two well-established models, one developed by R.N. Wenzel and one by A.B.D. Cassie and S. Baxter, are typically used to describe the water dewetting behavior on a rough surface. The researchers believe that their results are more consistent with the second model, which predicts a decrease in contact angle with bead diameter for the sizereduced beads in agreement with their results, whereas the first model predicts an increase in contact angle with bead diameter. The researchers also measured water-contact angles for several closepacked arrays formed with bead diameters from $270 \mathrm{~nm}$ to $690 \mathrm{~nm}$. The measured contact angle was $131^{\circ} \pm 2^{\circ}$, compared with a prediction of $141^{\circ}$ degrees for the Wenzel model and $133^{\circ}$ for the Cassie and Baxter model. The researchers said that for such nanostructured surfaces, the Cassie and Baxter model works better, with the exception of a few discrepancies. One is the difference in water-contact angle between single- and double-layer arrays. In contrast to the assumption that only the top layer affects contact angle, double-layer PS arrays have even higher contact angles than single-layer arrays, which can be explained by the defects formed during the nanosphere lithography process. Two more discrepancies are the surface fine structures caused by inhomogeneous oxygen etching and the imperfect spherical shape of the etched PS beads.

Furthermore, the measured water-contact angle hysteresis is rela-

\section{Know the Ups and Downs ...}

Steep slope

+ Big height difference

+ Large scan field

The LSM 5 PASCAL confocal laser scanning microscope broadens your microscopic horizons.
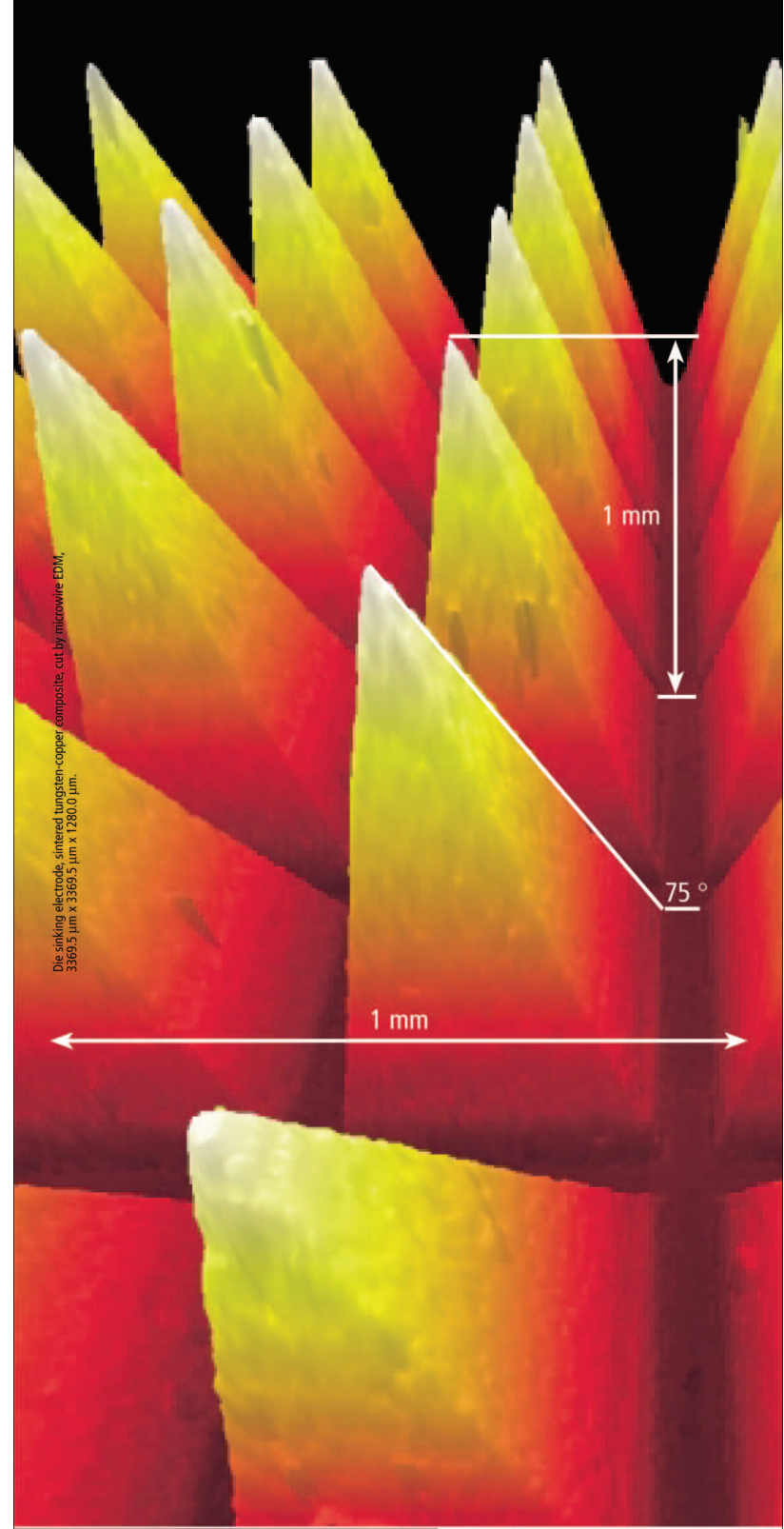

Highly resolved images.

Simple and efficient.

Non-destructive.

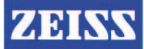

www.zeiss.com/lsm-mat

We make it visible. 
that you thought did not exist. .

\section{D MODELING} solutions

S3D Modeling software is a unique scientific tool for 3-dimensional modeling of granular media.

S3D Modeling product family provides comprehensive visual analysis and wide range of quantitive characteristics of the granular structures.

Nanofilms

Coatings

Powders

Ceramics

Composites

Refractories ****

Grain structure

Pore structure ***

Packing

Sintering

and more...

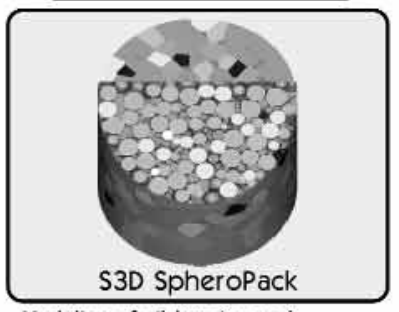

Modeling of oil-bearing rock

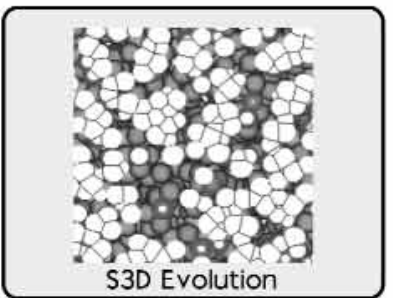

Modeling zonal segregation during the disperse powders sintering

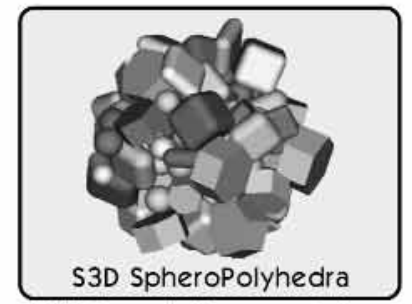

Multiphase polydisperse central packing of particles with varied morphology

wWw.smartimtech.com

(3) SMART IMAGING TECHNOLOGIES Tel: US: (877) 280.11.00 (toll-free) Tel: International +1 (713) 589.32.16 E-mail: info@smartimtech.com

For more information, see http://advertisers.mrs.org tively large, which can be attributed to the well-ordered nature of the system, according to the researchers. Contactangle hysteresis is due to the fact that the contact angle of water advancing on a structured surface can be different than the contact angle of water receding on the same surface.

\section{EKATERINA A. LITVINOVA}

\section{Molecules Self-Assembled on Nanowire FETs Serve as Multilevel Memory Element}

With Si-based memory technologies reaching their physical and technical limitations, researchers have focused on molecular memory devices in order to overcome these limitations. Such devices employ a method of storing charges at discrete reduction and oxidation states of molecules, with the possibility of storing multiple bits per cell. Due to the extremely small size of such technologies on the order of single molecules and the ability to self-assemble the molecular layers, this method could lead to extremely highdensity storage devices far beyond the capabilities of Si-based memories. In the March 15 issue of Applied Physics Letters, C. Li and C. Zhou of the University of Southern California, W. Fan of NASA Ames Research Center, and their colleagues have reported the development of multilevel molecular memory devices for storing up to three bits (eight levels) per cell. The device features a retention time of up to 25 days, which is much larger than in other molecular memory devices, and is realized based on nanowire field-effect transistors with self-assembled redox active molecules.

The basis of the device is semiconducting nanowire field effect transistors (FETs). Pulsed laser ablation was used to synthesize $n$-type $\operatorname{In}_{2} \mathrm{O}_{3}$ nanowires. The nanowires, which were $10 \mathrm{~nm}$ in diameter, were then deposited onto a wafer consisting of silicon covered by $100 \mathrm{~nm}$ of silicon dioxide. Source and drain electrodes were then patterned to form the FET. Finally, the nanowires were functionalized by immersing them in $0.5 \mathrm{mM}$ solutions of a molecule, which formed a monolayer onto the nanowire by self assembly. The $2 \mathrm{~nm}$ monolayer thickness of the self-assembled molecular layer was verified by ellipsometry.

The researchers studied different redox molecules, such as Fe-terpyridine, that varied either in metal ion $\left(\mathrm{Fe}^{2+}\right.$ and $\left.\mathrm{Co}^{2+}\right)$ or in the counter ion $\left(\mathrm{Cl}^{-}\right.$or $\left.\mathrm{PF}_{6}{ }^{-}\right)$. Writing and reading operations were accomplished by using gate voltage pulses and current sensing, respectively. The device can store multiple bits within the same single memory cell, instead of relying on storing single bits per unit. Such techniques have received increasingly more attention from semiconductor industry in recent years.

The research team estimated that the device features an extremely high storage density of $40 \mathrm{Gbits} / \mathrm{cm}^{2}$. An important advantage from the viewpoint of semiconductor processing is that the manufacture of this device is simpler than that of the classical silicon flash memory, since it involves only one step of photolithography and metallization. The new device also has strong advantages with respect to power consumption compared to silicon technology. The researchers propose that recent advances in nanowire assembling could be utilized to generate memory arrays.

MARKUS J. BUEHLER

\section{FOR MORE RESEARCI NEWS ON MATERIALS SCIENCE . . . \\ ... access the Materials Research Society Web site:}

www.mrs.org/gateway/ matl_news.html

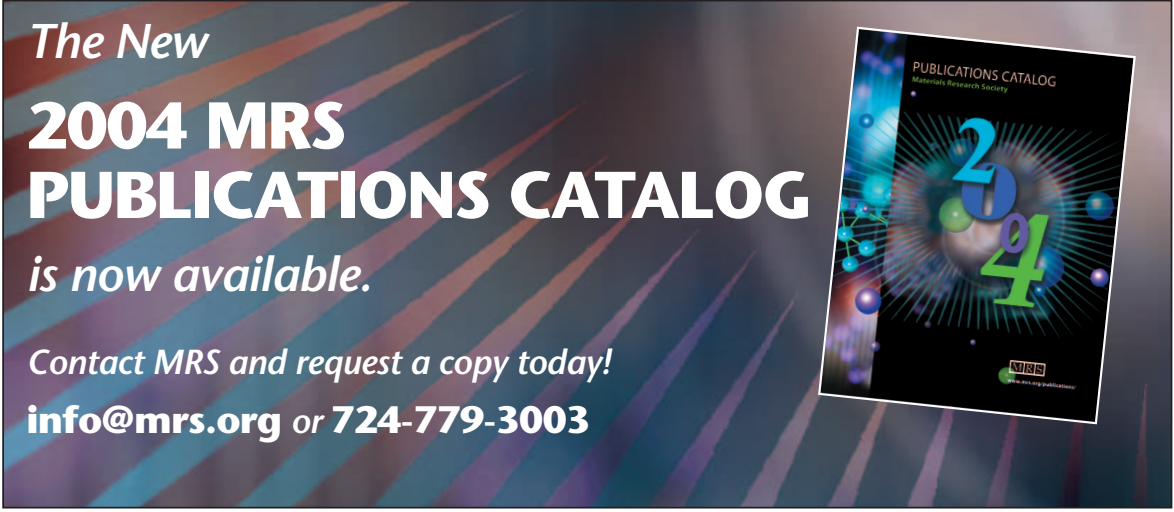

